Iustitia Socialis. Revista Arbitrada de Ciencias Jurídicas.

Año V. Vol. V. N9. Julio - Diciembre 2020

Hecho el depósito de Ley: FA2016000064

ISSN: 2542-3371

FUNDACIÓN KOINONIA (F.K). Santa Ana de Coro, Venezuela;

Tatiana Daniela Gárate-Osorio; Juan Carlos Erazo-Álvarez; Cecilia Ivonne Narváez-Zurita; Camilo Emanuel Pinos-Jaén

http://dx.doi.org/10.35381/racji.v5i9.718

\title{
Vulneración de los Derechos Humanos por la aplicabilidad de la norma en privados de libertad
}

\section{Violation of Human Rights by the applicability of the norm in deprived of liberty}

\author{
Tatiana Daniela Gárate-Osorio \\ tatiana.garate@psg.ucacue.edu.ec \\ Universidad Católica De Cuenca, Cuenca \\ Ecuador \\ https://orcid.org/0000-0001-9363-9759 \\ Juan Carlos Erazo-Álvarez \\ jcerazo@ucacue.edu.ec \\ Universidad Católica de Cuenca, Cuenca \\ Ecuador \\ https://orcid.org/0000-0001-6480-2270 \\ Cecilia Ivonne Narváez-Zurita \\ inarvaez@ucacue.edu.ec \\ Universidad Católica de Cuenca, Cuenca \\ Ecuador \\ https://orcid.org/0000-0002-7437-9880 \\ Camilo Emanuel Pinos-Jaén \\ cpinosj@ucacue.edu.ec \\ Universidad Católica de Cuenca, Cuenca \\ Ecuador \\ https://orcid.org/0000-0002-0934-8471
}

Recibido: 05 de abril de 2020

Revisado: 30 de abril de 2020

Aprobado: 20 de mayo de 2020

Publicado: 08 de junio de 2020 


\section{RESUMEN}

El objetivo de la investigación fue determinar la vulneración de derechos humanos por la inaplicación de las normas que regulan la protección de los derechos de las personas privadas de la libertad, en el acceso a la salud, alimentación y buen trato en el Centro de Rehabilitación Social Turi. Se abordó una tipología documental - bibliográfica jurídica. Como resultado de la investigación se identificó la vulneración de los derechos constitucionales a la alimentación, salud y buen trato; lo cual denota, la inobservancia de la Constitución de la República del Ecuador respecto a los artículos 32, 13, 51. Concluyéndose que si existiera un control y un trabajo adecuado de todos los funcionarios y autoridades se frenaría un buen porcentaje de las mafias existentes y las personas privadas de libertad incluso podrían aportar con su trabajo a nuestra sociedad.

Descriptores: Derechos humanos colectivos; política de la salud; derecho constitucional; política social; rehabilitación. (Palabras obtenidas del Tesauro UNESCO)

\section{ABSTRACT}

The objective of the investigation was to determine the violation of human rights by the non-application of the norms that regulate the protection of the rights of persons deprived of liberty, in access to health, food and good treatment at the Center for Social Rehabilitation Turi. A documentary - bibliographic - legal typology was addressed. As a result of the investigation, the violation of the constitutional rights to food, health and good treatment was identified; which denotes the non-observance of the Constitution of the Republic of Ecuador with respect to articles 32, 13, 51. Concluding that if there were adequate control and work of all officials and authorities, a good percentage of the existing mafias would be stopped and Persons deprived of liberty could even contribute their work to our society.

Descriptors: Collective human rights; health policy; constitutional law; social policy; rehabilitation. (Words taken from the UNESCO Thesaurus)

\section{INTRODUCCIÓN}

Los Derechos Humanos (DH) son inherentes a las personas por el sólo hecho de tener la condición de ser humano, sin ningún otro requisito. Estos derechos son irrevocables, inalienables, intransmisibles, irrenunciables y universales, cuya aplicación es directa y de cumplimiento inmediato. En este sentido, la presente investigación trata sobre la violación de los $\mathrm{DH}$ de las personas privadas de la libertad (PPL), en el Centro de 
Rehabilitación Social Turi, por cuanto, los derechos humanos de los PPL, en el Ecuador, tienen una atención prioritaria como grupo vulnerable según el artículo 35 de la Constitución de la República del Ecuador.

En función de lo expuesto supra, la investigación se realizó en el Centro de Rehabilitación Social (CRS) de Turi, en la que se investigó si se han vulnerado los derechos humanos por la falta de atención médica, alimentación adecuada, maltrato y otras vulneraciones; a partir del cual se crea un ambiente de violencia en el CRS; lo cual, no se orientaría a una rehabilitación social adecuada de las personas tal como garantiza nuestra Constitución de la República del Ecuador (CRE). Es necesario identificar y demostrar la existencia de vulneración de los derechos humanos.

Consecuentemente, se pretende demostrar el irrespeto generado a las PPL, mediante actos que vulneran las disposiciones constitucionales y estándares internacionales de derechos humanos, con lo cual se tomen las medidas correctivas para evitar la inobservancia, vulneración o menoscabo de los derechos en los CRS. En este caso, la investigación se focalizó en uno de los sectores sociales susceptibles de vulneración de los derechos fundamentales. La CRE en su artículo 426 establece que todas las personas deben respetar las normas constitucionales e internacionales en cuanto tenga relación con los derechos humanos, es decir las autoridades y funcionarios deben aplicar directa e inmediatamente normas tendientes a la protección de los derechos humanos, por cuanto, lo contrario implicaría el detrimento o la regresividad de los derechos (Constitución de la República del Ecuador, 2008).

La presente investigación, propone la adopción de medidas administrativas para la garantía y protección de los derechos humanos, con lo cual, se observe lo dispuesto en la Constitución respecto a las PPL y la rehabilitación acorde a sus necesidades y capacidades, la misma que servirá para que puedan desempeñar actividades lícitas al ser reinsertados a la sociedad. En virtud de los antecedentes expuestos el problema de la presente investigación es: ¿Cómo establecer la existencia de la vulneración a los derechos de las PPL en función de la salud, alimentación y buen trato?, a partir del cual el objetivo es analizar los efectos de la inaplicación de las normas que regulan la 
protección de los derechos de las PPLS, que permita el acceso a la salud, alimentación y buen trato en el Centro de Rehabilitación Social Turi.

\section{Referencial teórico}

\section{La Vulneración de los Derechos Humanos de las Personas Privadas de la Libertad (PPL).}

La armonía de los derechos humanos, son esenciales para tener una vida digna. Estos son inherentes a la especie humana con características de irrevocables, inalienables, intransmisibles, irrenunciables, universales. En otras palabras, los derechos humanos constituyen cimientos para una vida digna. No deben ser meras aspiraciones, por lo tanto, el rol del Estado consiste en garantizar y promover la protección de los derechos y no permitir su vulneración. Cabe indicar, que la dignidad humana depende del respeto de estos derechos. (Amnistía Internacional, 2014).

En el mismo orden de ideas, para cierto sector de la doctrina, es claro que los derechos humanos son universales, supra-estatales, es decir, para todos sin distinción alguna o que cumplan condiciones para poder ejercerlos, es por eso, que a ninguna persona se le puede negar estos derechos y deben estar vinculados y subordinados al Estado. Estos derechos deben estar sancionados positivamente para que tengan existencia en el ordenamiento jurídico de cada Estado; estos no son alienables menos aun negociables (Ferrajoli, 2001).

En líneas generales, estos derechos son de inmediata aplicación por ser supraestatales, que, en efecto, el Estado tiene que aplicarlos y no vulnerarlos, como ya se dijo en líneas anteriores tanto a los ciudadanos como a las PPL. Para poder proteger dichos derechos, es necesario que se encuentren en la normativa para que puedan ser aplicados directamente y sin ninguna excusa, siendo normativamente de todos, es decir, inherentes a cada una de las personas; sin duda no hay ninguna distinción entre las PPL y las que gozan de libertad en una sociedad, claramente las dos tienen los mismos derechos fundamentales y hay que respetarlos, con ciertas suspensiones (Salinas-Herrera, Narváez-Zurita, Vázquez-Calle, \& Erazo-Álvarez, 2020). 
Del mismo modo, los derechos humanos son reconocidos y calificados universalmente porque son totalmente fundamentales para que los seres humanos tengan una vida adecuada, considerando que estos derechos pertenecen a las personas inclusive antes de su nacimiento y, en caso de dudas sobre su aplicación, va a prevalecer la interpretación que más favorezca a la protección de las personas y a los derechos que son inherentes (Casar-Hernández, 2008).

En efecto, los derechos humanos son derivados de la dignidad inherente a todas las personas, sin distinción alguna; recalcando que cuando se pierde la libertad se sigue manteniendo la calidad de ser humano, calidad que servirá al momento de su inserción en la sociedad y su acoplamiento. Las PPL, deben ser tratadas como humanos y no como enemigos del Estado, para ello se proveerá una buena alimentación, salud, sin torturas e intimidaciones, debiendo los funcionarios de dichos centros trabajar de una manera adecuada, protegiendo los derechos humanos de estas personas, dándoles un trato igualitario y sin excusa para vulnerarlos. Ahora bien, ningún recluso debería temer por su seguridad y la de su familia, las autoridades deben proteger a este grupo de personas que son vulnerables como manifiesta el artículo 35 de la CRE siendo el Estado responsable de las PPL.

Por lo antes descrito, toda PPL tiene derecho a ser tratada con el respeto debido, cuidando su integridad y dignidad que el ser humano se merece; por cuanto, el irrespeto de los mismo desprotege la dignidad por la inobservancia de derechos, principios y reglas. Atendiendo a estas consideraciones se debe reflexionar si los CRS son un laboratorio de lo social, sabiendo que en estos sitios se puede demostrar la estructura social nacional, en donde el Estado muestra sus límites y su capacidad de organizar, evidenciando que existe un desinterés en el sistema penitenciario, al no haber un control sobre la vulneración de los derechos a las PPL, a pesar de que el Estado es responsable de mantener un sistema adecuado sin deficiencias operativas que vulneran sistemáticamente los derechos (Stieppel, 2013).

En los CRS, la incidencia de violación de los derechos humanos es muy alta y sobre todo frecuente, que hace que la vida de los internos sea muy dolorosa; es difícil pensar 
que no se cumple lo que indica claramente la CRE en su artículo 426, ya antes mencionado, sobre el cumplimiento y aplicación inmediata de las normas tanto nacionales e internacionales sobre los derechos humanos (Constitución de la República del Ecuador, 2008).

El Estado ecuatoriano al ser garante de los derechos fundamentales de las PPL debe gestionar las políticas públicas necesarias, que garantice los derechos por ejemplo, cuidando la seguridad interna, dando atención médica, una alimentación saludable y un trato digno en los CRS; de esa manera, ayudaría a la protección de estos derechos humanos, consiguiendo que las personas privadas de la libertad cumplan su condena de dignamente; siendo los derechos humanos y sus garantías, el mejor instrumento para expresar los intereses y necesidades de millones de personas.

Podría pensarse que las PPL, al perder la libertad temporalmente, pierden también sus derechos; todo lo contrario, si bien es cierto se suspenden o restringen temporalmente derechos, eso los convierte en personas vulnerables y la necesidad de garantizarlos. Sin duda, siguen siendo seres humanos y el derecho que perdieron temporalmente es la libertad, por mencionar alguno.

En concordancia con lo anteriormente descrito, la CRE en su artículo 35 señala que el Estado protegerá a las personas vulnerables recibiendo atención prioritaria y especializada tanto en lo público como en lo privado (Constitución de la República del Ecuador, 2008). Dentro de este grupo vulnerable están las personas privadas de la libertad, por lo que los funcionarios deben cumplir esta norma generando políticas administrativas que garanticen, los derechos humanos en favor de las PPL. En este mismo sentido el artículo 10 numeral 3 y 7 de la CRE manifiesta que los derechos humanos son de inmediata aplicación por y ante cualquier servidor público de oficio o a petición de parte debe aplicarlos y protegerlos, estos derechos no excluirán a los demás derechos que se deriven de una dignidad humana (Aldana-Zavala \& Isea, 2018a).

De la misma manera en su artículo 201 y 202 determina que el Sistema Nacional de Rehabilitación Social tendrá como finalidad la rehabilitación integral de las personas sentenciadas penalmente para reinsertarlas en la sociedad, así como la protección de 
las PPL y la garantía de sus derechos, garantizando evaluar que se cumpla con eficacia las políticas públicas de los CRS, para garantizar el objetivo de la rehabilitación (Constitución de la República del Ecuador, 2008).

De igual forma, el artículo 674 del Código Orgánico Integral Penal (COIP), establece que el Sistema de Rehabilitación debe garantizar el cumplimento de sus fines mediante un Organismo Técnico, cuyas atribuciones son evaluar la eficacia de las políticas del sistema que administran los CRS y fijar los estándares de cumplimiento sobre los fines y objetivos de estos centros (Constitución de la República del Ecuador, 2014).

Como complemento, el Reglamento del Sistema Nacional de Rehabilitación Social del Ministerio de Justicia en su artículo 1 señala que esta normativa se va a regir en los principios constitucionales y en los instrumentos internacionales de derechos humanos, aplicando dichas políticas de manera progresiva; en este sentido, el artículo 11 indica claramente en su objetico la capacitación de una forma permanente a todo el personal penitenciario, para que conozcan sobre todas las normativas vigentes y sobre la protección de los derechos humanos, teniendo la finalidad de fomentar la igualdad y no discriminación a las PPL, para que dentro de los CRS tengan una vida digna y garantizando el respetando sus derechos (Ministerio de Justicia Derechos Humanos y Cultos, 2016).

En concordancia con lo indicado, para que se cumpla con el objetivo de los CRS y se protejan los derechos humanos con un adecuado control, la presidencia de la República del año 2014, mediante decreto № 365, crea el Organismo Técnico del Sistema Nacional de Rehabilitación Social, integrado por: El Ministro de Justicia, Derechos Humanos y Cultos, quien lo presidirá; El Ministro de Salud Pública; el Ministro de Trabajo; El Ministro de Educación; El Ministro de Inclusión Económica y Social; El Ministro de Cultura y Patrimonio; El Ministro del Deporte; y, El Defensor del Pueblo, autoridades que tienen la obligación de hacer cumplir toda la normativa que sea respecto de los derechos humanos de las personas privadas de la libertad (Presidencia de la República, 2014). 
En este mismo sentido mediante decreto 1317 se responsabiliza al Ministerio de Justicia y Derechos Humanos, actualmente llamada, Secretaría de Derechos Humanos, de coordinar y controlar que se cumplan las políticas del estado y dar el seguimiento necesario (Presidencia de la República, 2008). Concordante con lo antes expuesto, se expidió el Reglamento para el funcionamiento del Organismo Técnico del sistema nacional de Rehabilitación, teniendo el objeto de establecer el marco orgánico y normativo de los centros de conformidad con la CRE, además, evaluará la eficacia de las políticas que han establecido los CRS (Organismo Técnico de Rehabilitación Social, 2018). Es evidente la existencia de normativa, sin embargo, se necesita un órgano encargado de aplicar y controlar lo dispuesto, caso contrario, son buenas intenciones que no se reflejan en la realidad.

Cabe recalcar, que la CortelDH ha señalado que los derechos humanos no son una opción ni una alternativa, al contrario, por ser exigibles, son de directa e inmediata aplicación por y ante cualquier autoridad publica. El Estado es el que debe asumir la responsabilidad de tomar todas las iniciativas y medidas que sean necesarias para que garantice a las PPL, las condiciones adecuadas y que dentro de su rehabilitación gocen de los derechos humanos y que bajo ningún motivo o circunstancia sean vulnerados (Corte Interamericana de Derechos Humanos, 2020).

Las entidades públicas y en especial las encargadas de la administración de justicia, están obligados a respetar estos derechos y de ninguna manera podrán inobservarlos, por cuanto podria afectarse la calidad de vida de las personas, más aún, si están privadas de su libertad. Ahora bien, los artículos 417 y 424 de la CRE señalan claramente que en caso de los derechos humanos se aplicará los principios pro ser humano, de aplicación directa y de cláusula abierta, además los Tratados Internacionales sobre derechos humanos que estén ratificados por el Estado, van a prevalecer sobre cualquier otra norma jurídica (Constitución de la República del Ecuador, 2008).

En este sentido, el Ecuador firmó y ratificó la Convención Americana sobre de Derechos Humanos, por lo tanto, los derechos reconocidos en la misma son de aplicación directa, 
así en la CRE no estuviere, esto se da por el principio de subsidiariedad o complementariedad; siendo así, estamos obligados a la aplicación inmediata sin que, la falta de conocimiento de la normativa, sirva como justificativo para la vulneración de los derechos. Los funcionarios y administradores de justicia (juezas, jueces) están en la obligación, de realizar todo lo que sea necesario para que no se vulneren los derechos, más aún, si estas quienes fueran afectada estén en un estado de vulneración (AldanaZavala \& Isea-Argüelles, 2018b).

Dentro de este orden de ideas, la Declaración Universal de los Derechos Humanos determinados por la Organización de las Naciones Unidas (ONU) describe detalladamente aquellas libertades, facultades, instituciones o reivindicaciones relativas a los bienes primarios o básicos que necesitan todas las personas, por el simple hecho de su condición humana, garantizando una vida digna y plena; sin distinción de raza, sexo, religión, pensamiento político, idioma o condición económica (Asamblea General de las Naciones Unidas, 1948).

Claramente estos derechos no son renunciables y deben ser respetados, para que estos derechos no sean vulnerados los Estados deben generar políticas administrativas que garanticen la aplicación de los derechos humanos, sobre todo a las PPL que el Estado es responsable de ellas, dicho de otro modo, los Derechos Humanos son muy necesarios en la vida de cualquier persona, pues estos garantizan una vida digna, permite la igualdad y justicia, desprotegerlos, sería inhumano.

\section{Los Derechos Humanos como garantía de una vida digna}

Como ya se mencionó, la tortura no solo deriva en el maltrato físico, sino también en el psicológico. La tortura se da cuando una autoridad pública con el objetivo de obtener alguna información, realiza actos crueles que causan dolor, a esta tortura la llaman tortura judicial cuando estos maltratos son causados para llegar a la culpabilidad del acusado. De este modo la tortura es un acto indebido que vulnera derechos humanos de una manera denigrante aprovechándose de la vulnerabilidad de las PPL, las mismas que no pueden defenderse y lo único que les toca es soportar estos maltratos por temor 
a las represalias, resulta claro, que la tortura constituye una vulneración grave a los derechos humanos, tanto así que desde el año de 1948 la comunidad internacional prohibió la tortura y otros tratos crueles, inhumanos o degradantes en su artículo 5, manifestando que ninguna persona puede ser sometida a torturas (Asamblea General de las Naciones Unidas, 1948).

Por otra parte, la tortura fue incorporada como una violación grave a los derechos humanos y de sanción obligatoria para los Estados, en este sentido el artículo 7 del Pacto Internacional de Derechos Civiles y Políticos, manifiesta que nadie puede ser sometido a torturas (Naciones Unidas, 1976). A pesar de que existe una normativa muy clara sobre la prohibición de la tortura, sigue habiendo estos actos denigrantes sin importarles la normativa vigente, esto se debe que cuando las personas han perdido su derecho a la libertad, los funcionarios de los CRS y la sociedad ya no los ven como seres humanos y piensan que no tienen los demás derechos, por eso algunos funcionarios abusan de su condición para maltratarlos y obtener información que necesitan (Rodas-Cordero, Erazo-Álvarez, Pinos-Jaén, \& Narváez-Zurita, 2020).

Ha quedado demostrado que la tortura está prohibida, tal es el caso, que el primer instrumento que define la tortura en su artículo 1 es la Convención contra la Tortura y Otros Tratos o Penas Crueles, que indica que la tortura es cualquier acto que una persona que está dentro de sus funciones públicas, que intencionalmente cause a otra ya sean estos maltratos físicos o psicológicos con un fin de obtener una confesión (Naciones Unidas, 1987).

De igual manera la Constitución de la República del Ecuador, en el artículo 66, numeral 3 literal c, prohíbe las penas crueles, las torturas y todo trato cruel, inhumano o degradante (Constitución de la República del Ecuador, 2008). En este mismo contexto, el Plan Nacional de Derechos Humanos del Ecuador de 1998, en el artículo 4 indica que se debe lograr que los sistemas de detención, investigación y penitenciario destierren las prácticas de tortura, maltratos físicos y morales como mecanismo de investigación y castigo (Presidente Constitucional Interino de la República, 1998). Como se evidencia hay normativa que prohíbe la tortura, es por eso que no se debe permitir 
de ninguna manera estos actos y el Estado debe ser el primero que proteja este derecho a no ser torturado y analizar todo lo que ocurre con las PPL y dar soluciones efectivas, por cuanto, tienen derecho a tener un buen trato por citar dentro de su rehabilitación.

Siendo las cosas así, es importante mencionar que en el Juicio № 01283201604915G, las PPL reflejan sobre la torturas que se dan dentro del CRS Turi, testificando que les golpeaban entre 15 personas y a eso lo llamaban el camionetazo; además, se les golpeaba con tubos, provocando lesiones graves en su cuerpo y se promediaba agresiones de hasta 30 veces. Se dice que fueron ingresados en la ducha para baños de agua fría y les pasaban cables de electricidad; se evidencia claramente en los relatos, que las PPL han vivido una pesadilla de dolor tanto físico como psicológicos (Tribunal de garantías penales con sede en el cantón Cuenca, 2019).

Estos relatos constan en la audiencia de dictamen fiscal llevada a cabo el 07 de septiembre de 2017, en esta diligencia se demostró con evidencias que sustentaron las versiones rendidas. Dentro de este proceso judicial las declaraciones que dan las personas privadas de libertad son impactantes, dolorosas, demostrando que la vida que llevan en el Centro de Rehabilitación es totalmente brutal y degradable, Ilena de humillaciones y maltratos que van en contra de la dignidad humana. No es posible imaginar que, dentro de un CRS, exista todas estas vulneraciones a seres humanos, que ya por el simple hecho de perder su libertad están en una situación difícil, más aún cuando se vulneran los demás derechos que son totalmente necesario para tener una vida digna dentro de su rehabilitación, en este sentido si hubiera control de las políticas internas, no habría tanta vulneración a estos derechos tan esenciales.

En lo que respecta al derecho de acceso al servicio de salud, este se constituye en un derecho fundamental, en favor de las personas privadas de la libertad ya que la vida está en juego, en líneas generales me refiero al derecho que tiene un privado de la liberta a tener una atención médica cuando lo necesiten de manera oportuna y además tener todas las precauciones sanitarias para prevenir enfermedades, las PPL también tienen este derecho y no se les debe vulnerar por ninguna causa. Hay casos que tienen 
que soportar un dolor porque no tienen médicos o porque no pueden salir para recibir atención en centros hospitalarios externos para ser atendidos, o lo que es peor porque los funcionarios no les ayudan con el trámite para que puedan acudir a un médico, incluso cuando tienen enfermedades como es la diabetes, cáncer, gastritis, entre otras, no tienen el tratamiento adecuado, lo que produce que se apeore su enfermedad (Baculima-Llivisaca, Narváez-Zurita, Trelles-Vicuña, \& Erazo-Álvarez, 2020).

Dentro de este orden de ideas, el derecho a la salud se encuentra determinada en el artículo 32 de la CRE, señalando que, el Estado garantiza este derecho generando políticas públicas, sociales económicas culturales etc. protegiendo el acceso continuo de una manera equitativa, universal, solidaria, interculturalidad, eficiencia sin ningún tipo de discriminación (Constitución de la República del Ecuador, 2008). Las PPL se encuentran como grupo vulnerable, y tienen el derecho de atención prioritaria, sin que haya prohibición de este derecho y se restrinja el acceso a la salud; por cuanto al estar privados de la libertad, dependen de los funcionarios que trabajan en CRS, los mismos que deben realizar todo lo que sea necesario para que una persona privada de la libertad pueda acudir a los dispensarios médicos o en casos ya mayores, a los hospitales públicos.

La salud es un derecho humano fundamental y si no se tiene acceso a este derecho, no se lo está garantizando, es por ello, que el Estado debe proteger y responder por la prestación del servicio, bien sea de parte pública o con convenios privados, de esa manera demuestra una democracia y equidad en una nación (Barrera, 2018). Es decir, los Estados tienen la responsabilidad que todas las personas, privadas o no de la libertad, tengan derecho a su acceso salud, lo cuan tienen la obligación de realizar medidas necesarias tanto sanitarias como sociales adecuadas para que a nadie se le vulnere (Asamblea Mundial de la Salud, 1946).

En el mismo enfoque dentro de un trabajo de investigación para obtener el título de Magister en salud pública se sostiene que existen varios estudios en algunas partes del mundo que demuestran que dentro de los Centros de Rehabilitación la salud de las PPL es más deteriorada que en la población que están libre, debido que, las personas que 
se encuentran privadas de la libertad no tienen oportunidad adecuada para acceder a todos los servicios de salud, ya que, en estos centros más se preocupan por el orden, el control y la disciplina. (Pacheco, 2016). A este respecto, la PPL es evidente que por su condición tienen una salud deteriorada inclusive porque no tienen una buena alimentación, los protocolos de higiene necesarios y acceder a los centros médicos se les dificulta, por lo que dependen de terceras personas, además que dentro de los CRS no hay un sistema adecuado de dispensarios médicos para poder atenderles.

La Corte Constitucional ya se ha referido sobre el tema y, citando a la la Corte Interamericana de Derechos Humanos (CorteIDH), ha manifestado que los Estados deben proporcionar a las revisiones médicas y estas deben ser continuas, dándoles los tratamiento que necesiten; es decir, el Estado debe garantizar el derecho de salud que incluye no solo atención médica si no psiquiátrica y odontológica, además que exista personal médico que trabaje imparcialmente, también deben tener acceso a la medicación gratuita y deben ser de acuerdo a cada caso, igualmente indica en la sentencia que se debe implementar programas relacionados a la salud, inmunización, prevención y tratamiento de enfermedades infecciosas, endémicas; concluyendo que las PPL pertenecen a grupos vulnerables o de alto riesgo. (Corte Constitucional del Ecuador, 2019).

De esta manera la Corte Constitucional al recoger el precedente marcado por la CortelDH, desarrolla el derecho a la salud de las PPL, quienes además tienen derecho a revisiones periódicas tanto médicas como psicológicas, siendo obligación del Estado, garantizar generar políticas públicas internas para la eficacia de este derecho. En el mismo contexto, la Corte Constitucional relaciona a la integridad física con el derecho a la salud, con lo cual se genera la obligación del Estado de garantizar la atención médica para que tenga un mejor nivel de vida, incluso, al vulnerar el derecho a la salud, puede tener un impacto muy grande en el derecho a su integridad física, pudiendo las personas privadas de la libertad utilizar la acción constitucional de habeas corpus.

Sobre las bases de las ideas antes expuestas, podemos arribamos a la conclusión de que todas las personas tenemos derecho a la salud, sin embargo, existe una especial 
protección a las personas privadas de la libertad en CRS, tanto es así que, por el hecho de perder la libertad, esto no significa que se pierde el derecho a la salud, en consecuencia, no se puede tolerar que el encarcelamiento sea un signo de enfermedad y padecimiento (Corte Constitucional del Ecuador, 2019). En este sentido, las autoridades estatales deben formular protocolos de atención, para que las PPL puedan asistir a dispensarios médicos y psicológicos, los cuales sean seguros y se encuentren en buen estado y al alcance de las PPL, esto con el objeto de evitar la vulneración del derecho a la salud.

Asimismo, es importante analizar el derecho a la alimentación que sin duda es esencial para la vida de un ser humano. Es un derecho fundamental e irrenunciable, más aún las PPL que se encuentran a cargo del Estado por su condición. El Estado es responsable de las PPL y deber tener políticas para que no se vulnere este derecho. De hecho, la alimentación debería definirse como un derecho universal que toda persona debería tener, este derecho se debería garantizar mediante modelos de producción sostenibles en un entorno de salubridad. Este derecho es fundamental para la vida y ligado a la dignidad humana, que no solo es una obligación moral si no también es jurídica de conformidad con las normas internacionales por lo que los estados deben organizar las medidas que sean necesarias para proteger este derecho, existe una estrecha relación entre la salud y la alimentación puesto a que si hay una mala alimentación las personas no tendrían una buena salud (Carretero-García, 2018).

De esta manera la Declaración Universal de Derechos Humanos indica que toda persona tiene derecho a una alimentación adecuada para una vida digna (Asamblea General de las Naciones Unidas, 1948). Asimismo, el Pacto Internacional de Derechos Económicos, Sociales y Culturales, en su artículo 11 reconoce una vida adecuada para las personas y su familia nombrando en el artículo la alimentación como parte esencial, indicando que los estados que formen parte del Pacto deben reconocer y proteger los derechos, además que toda persona debe estar protegida contra el hambre mediante políticas internas e internacionales (Naciones Unidas, 1976). 
Respecto al tema, con fecha 17 de octubre de 2018, se publicó un artículo sobre la calidad de la comida de los presos, investigación realizada el 19 de febrero de 2018, a través de la cual se dio a conocer la falta de vigilancia sobre la alimentación en los CRS, y se insta al Estado un mayor control a las empresas proveedoras para que cumplan con el servicio de una manera adecuada; además indica que, las tiendas que se llaman economatos, les obligan a depositar entre \$15 a \$ 40 cada semana para que puedan obtener otro tipo de comida, menciona que el estado determino un valor de $\$$ 30’383.631 para la alimentación en el año 2017 para los 55 centros, firmando un convenio con la empresa Lafattoria.

Sin embargo familiares de las personas privadas de la libertad indicaron que aún hay problemas serios en la alimentación y que los economatos solo buscan lucrar de los familiares, también, existen declaraciones de algunas personas PPL, quienes manifestaron que para poder comer tenían que tragar sin masticar, otra interna comenta que no se va olvidad a lo que le llamaban el sango de la muerte que era una comida en base de pescado dañado, por este tipo de alimentación muchas PPL obtienen otras enfermedades por la mala alimentación (El Telégrafo, 2018).

Es así que, el artículo 13 de la CRE establece que todas las personas tienen derecho a una alimentación sana y que el Estado protegerá este derecho (Constitución de la República del Ecuador, 2008). Evidentemente, el derecho a tener una alimentación sana es muy importante y se debe proteger con las medidas que sean necesarias; así mismo, el artículo 15 del Reglamento del Sistema Nacional de Rehabilitación Social señala que las PPL, señala que tienen derecho a las tres comidas diarias y la misma tiene que ser sana y con todos los cuidados sanitarios, a más de que los horarios deben ser adecuados, en caso de que una persona privada de la libertad por salud necesite otro tipo de dieta, no se le podrá negar y la máxima autoridad del centro debe velar por los derechos de las PPL (Ministerio de Justicia Derechos Humanos y Cultos, 2016). El derecho a la alimentación es esencial para la vida de las personas, la dieta debe ser adecuada y si las personas privadas de la libertad están a cargo del gobierno, deben 
recibir una alimentación sana; recordemos que son seres humanos que necesitan alimentarse adecuadamente y sobre todo con alimentos saludables.

\section{MÉTODO}

De conformidad con las aportaciones obtenidas, en la presente investigación, la metodología se basó en la modalidad de carácter descriptivo no experimental puesto que se realizó el estudio de las variables en el contexto natural de los sucesos, sin manipularlas, para su posterior análisis, síntesis y conclusiones (Reyes-Idrovo, Narváez-Zurita, Pozo-Cabrera, \& Erazo-Álvarez, 2020), mediante un diseño bibliográfico - documental (Hernández-Sampieri, Fernández-Collado, \& Baptista-Lucio, 2014), lo cual permitió generar un análisis del objeto de estudio con la finalida de lograr el objetivo propuesto por los investigadores, promoviendose un proceso analítico reflexivo, contribuyendo a la generación de la amplicación del estado del arte en relación al marco juridico cientifico vigente (Behar-Rivero, 2018).

La población de estudio estuvo conformada por 17 familiares de las Personas Privadas de Libertad en Centro de Rehabilitación Social Turi, a quienes se les aplicó una encuesta con la finalidad de conocer sus perspectivas sobre los derechos humanos de sus familiares privados de libertad, así mismo se analizaron teorías, leyes, procedimientos judiciales, jurisprudencias, como parte de la población de estudio, generándose mediante el análisis documental jurídico, la construcción de aportes reflexivos teóricos en el campo del derecho constitucional (Erazo-Álvarez \& NarváezZurita, 2020). 
Iustitia Socialis. Revista Arbitrada de Ciencias Jurídicas.

Año V. Vol. V. $N^{\circ} 9$. Julio - Diciembre 2020

Hecho el depósito de Ley: FA2016000064

ISSN: 2542-3371

FUNDACIÓN KOINONIA (F.K). Santa Ana de Coro, Venezuela;

Tatiana Daniela Gárate-Osorio; Juan Carlos Erazo-Álvarez; Cecilia Ivonne Narváez-Zurita; Camilo Emanuel Pinos-Jaén

\section{RESULTADOS}

A continuación, se presenta la tabla y la figura 1 con el resumen de los resultados obtenidos, clasificados según las variables definidas en el instrumento de investigación aplicado:

\section{Tabla 1}

Resultados de la encuesta aplicada a los familiares de los PPL del C.R. Turi.

\begin{tabular}{|c|c|c|c|}
\hline \multirow[b]{2}{*}{ Variable } & \multirow[b]{2}{*}{ Pregunta } & \multicolumn{2}{|c|}{ Resultado \% } \\
\hline & & Si & No \\
\hline & ¿Existe la vulneración del & & \\
\hline \multirow[t]{2}{*}{ Alimentación } & derecho de alimentación? & $100,00 \%$ & $0,00 \%$ \\
\hline & ¿Existe la vulneración del & & \\
\hline \multirow[t]{3}{*}{ Salud } & derecho de salud? & $100,00 \%$ & $0,00 \%$ \\
\hline & ¿Existe un buen trato a las & & \\
\hline & Personas privadas de la & & \\
\hline \multirow[t]{2}{*}{ Buen trato } & libertad? & $11,76 \%$ & $88,24 \%$ \\
\hline & ¿Considera que & & \\
\hline \multirow[t]{2}{*}{ Abuso físico } & abusadas físicamente? & $94,12 \%$ & $5,88 \%$ \\
\hline & ¿Considera son abusadas & & \\
\hline \multirow[t]{4}{*}{ Abuso psicológico } & psicológicamente? & $94,12 \%$ & $5,88 \%$ \\
\hline & ¿Las autoridades controlan & & \\
\hline & que no se vulneren los & & \\
\hline & derechos de las Personas & & \\
\hline Control & privadas de la libertad? & $94,12 \%$ & $5,88 \%$ \\
\hline
\end{tabular}

Fuente: Investigación de campo. 


\section{Resultados}

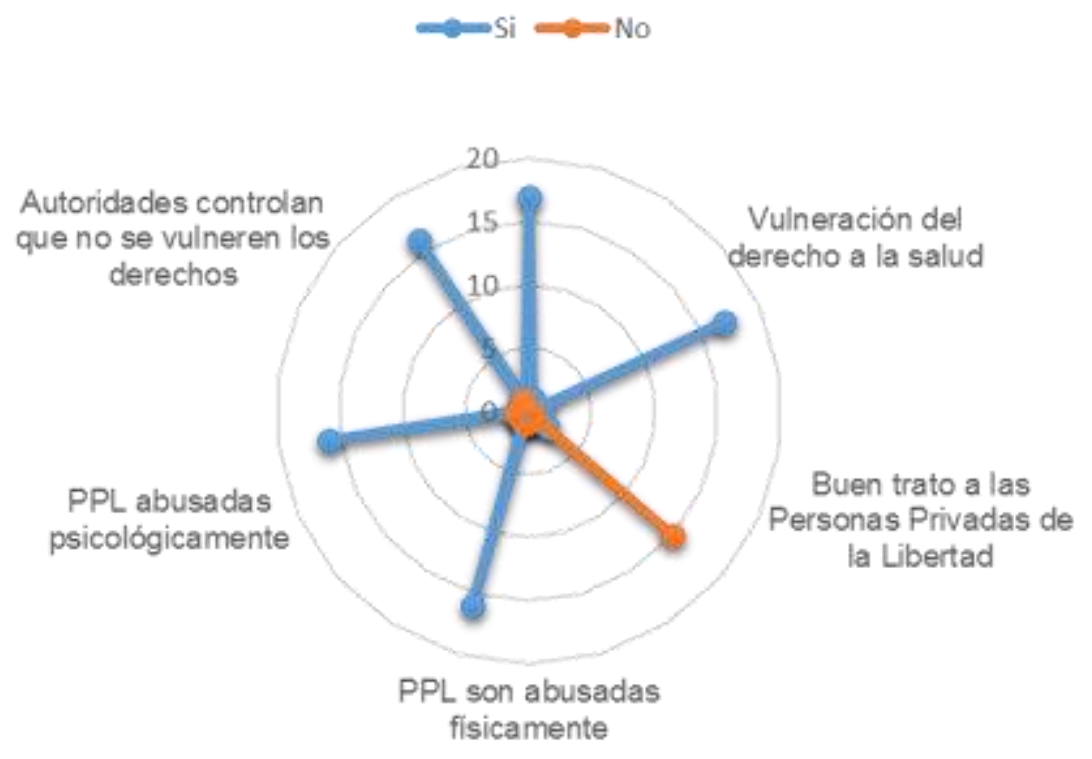

Figura 1. Representación gráfica de los resultados. Fuente: Investigación de campo.

En los resultados se puede destacar que los familiares de las personas privadas de la libertad coinciden en que al interior del Centro de Rehabilitación Social Regional Centro Sur Turi se vulneran los derechos de la alimentación, salud y buen trato a los PPL. Al respecto los encuestados afirman que sus familiares no tienen una adecuada alimentación debido a que la comida no es saludable, las porciones no son las adecuadas, no existe un plan alimenticio, los recursos del estado son insuficientes para la alimentación, dando como resultado afectaciones en la salud.

En cuanto al derecho de salud, se puede identificar que existe una vulneración, debido a que no se les atiende de manera oportuna, no hay la medicación necesaria, no tienen el personal médico suficiente, restringiendo su acceso oportuno siendo ellos personas vulnerables. De la misma manera la variable identificada al buen trato se refleja que existe maltratos tanto físicos como psicológicos, por el abuso de los guías con el pretexto de mantener el orden, tanto así que las personas privadas de la libertad tienen 
miedo a quejarse o reclamar por las represalias posteriores, por lo que optan por el silencio.

Esto se confirma de acuerdo a (INREDH, 2018) que señalan que estos casos se dan con cierta frecuencia y que demuestran la falta de humanidad de los funcionarios públicos del sistema penitenciario. Además, evidencia que el trato cruel, inhumano y degradante en las cárceles está al orden del día. Uno de los ejemplos más difundidos, es el caso de la cárcel de Turi en Cuenca donde más de 200 internos del pabellón de Medina Seguridad JC, en el año 2016, fueron víctimas de torturas cometidas por agentes de la Policía Nacional.

Estos casos de tortura y tratos crueles, inhumanos y degradantes reconocidos como tal en sentencias constitucionales, se agravó con el descubrimiento de una red de extorsión con participación de internos y administrativos del CRS Turi. Los hechos antes mencionados se los reconoció a través del Tribunal de Garantías Penales del Azuay como agresión por parte de los miembros de las unidades de Policía, que, aunque no se logró identificar sus rostros, se estableció la prisión para el jefe del operativo y los 36 de tropa (El Universo, 2020).

Todos estos maltratos se dan debido a que las autoridades no controlan adecuadamente el sistema. No existen mecanismos adecuados de prevención y posterior sanción al personal que vulnere los derechos antes mencionados. Son las autoridades las responsables de velar por los derechos humanos de las personas. De manera general se puede concluir que los datos levantados reflejan que a las personas privadas de la libertad se les vulneran estos derechos tan esenciales para una vida digna y que las autoridades competentes no realizan un control eficiente del cumplimiento de la normativa tanto nacional como internacional. 


\section{PROPUESTA}

En el Ecuador la CRE en el artículo 85, reconoce como garantía constitucional, las garantías de políticas públicas, las cuales tienen como objeto la obligación de desarrollar inter alia, Protocolos para hacer efectivos los derechos reconocidos en la CRE; razón por la cual, como se ha justificado en la presente investigación, se requiere de un Protocolo de Cumplimiento de la Normativa que proteja especialmente los derechos reconocidos en el artículo 51 de la CRE a favor de las PPL, el cual, contenga lo siguiente:

1.- Coordinación de la Secretaría de Derechos Humanos: Corresponde la coordinación con el Organismo Técnico del Sistema Nacional de Rehabilitación Social, los procedimientos, que permitan verificar el cumplimiento y respeto de los derechos humanos en los Centros de Rehabilitación Social (CRS).

2.- Capacitaciones sobre derechos humanos: La Defensoría del pueblo capacitará a los directores de los CRS de forma obligatoria. Esta capacitación será replicada por el director hacia los funcionarios y a las PPL en cada uno de los CRS.

3.- Control interno en los centros de rehabilitación: El departamento de control interno dentro de los centros de rehabilitación, verificará mensualmente las actividades que realizan los funcionarios. Este control debe ser notificado al director del Centro; quien, a su vez, podrá solicitar informes de oficio y emprender los correctivos necesarios.

4.- Control externo por parte de la Defensoría del Pueblo: El Defensor del Pueblo o su delegado, acudirán a los Centros de Rehabilitación para conocer de cerca el cumplimiento de los derechos humanos de las PPL, para lo cual, deberán entrevistarlos y emitir informes de los resultados. Los informes deberán ser comunicados a los directores de los Centros, quienes a su vez realizarán las correcciones pertinentes, de haberlas. El Defensor del Pueblo o su delegado, deben realizar el seguimiento del informe, para lo cual, frente a la inobservancia del mismo, se tomarán las acciones constitucionales o legales a las que haya lugar. 
5.- Sanciones por incumplimiento: En caso de incumplimiento de la normativa de protección a los Derechos Humanos, los directores y funcionarios del CRS, serán sancionados según corresponda.

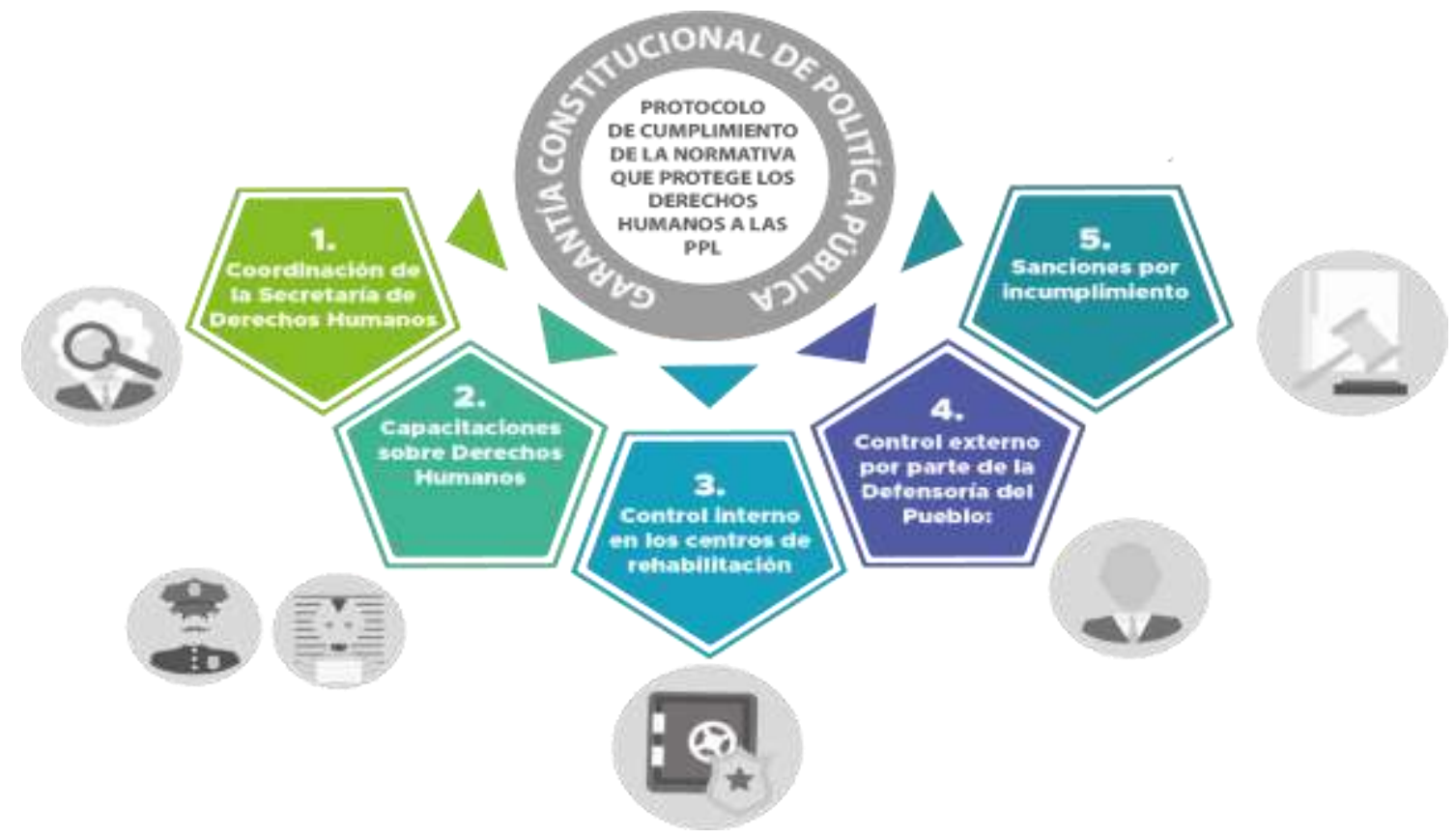

Figura 2. Estructura del protocolo propuesto.

La propuesta generada de acuerdo con la investigación, consiste en la elaboración de un protocolo de protección de derechos humanos para las personas privadas de la libertad, como garantía de política pública, el cual le corresponde coordinar a la Secretaría de Derechos Humanos conjuntamente con el Organismo Técnico del Sistema Nacional de Rehabilitación Social, estableciendo procedimientos que permitan verificar el cumplimiento y respeto de los derechos humanos en los Centros de Rehabilitación Social.

Para ello, además, se coordinará con la Defensoría del pueblo, diferentes capacitaciones sobre derechos humanos dirigidas a los directores de los CRS de forma obligatoria; quienes, a su vez, deberán replicar permanentemente en cada uno de los CRS que representan tanto a sus funcionarios y a las PPL. Para el cumplimiento del presente objetivo, se deberá crear el departamento de control interno en cada CRS, 
encargados de verificar el cumplimiento de los derechos humanos dentro del mismo. Para ello, emitirán informes mensuales al director del CRS, sin perjuicio que lo solicite de oficio. El director deberá emplear los correctivos necesarios de conformidad con la Constitución y la ley.

Por otra parte, en ejercicio de la garantía constitucional de tipo institucional, el Defensor del Pueblo o su delegado, acudirán a los Centros de Rehabilitación para conocer de cerca el cumplimiento de los derechos humanos de las PPL, para lo cual, deberán entrevistarlos y emitir informes de los resultados. Los informes deberán ser comunicados a los directores de los Centros, quienes a su vez realizarán las correcciones pertinentes, de haberlas. El Defensor del Pueblo o su delegado, deben realizar el seguimiento del informe, para lo cual, frente a la inobservancia del mismo, se tomarán las acciones constitucionales o legales a las que haya lugar. Finalmente, en caso de incumplimiento de la normativa de protección a los Derechos Humanos, los directores y funcionarios del CRS, serán sancionados según corresponda.

\section{DISCUSIÓN}

En el centro materia de la investigación existe claramente una vulneración al derecho de la salud, debido a que el acceso a un médico o a una casa de salud es muy limitado, no tienen la medicación necesaria, no tienen el personal médico suficiente, esta vulneración afecta de manera directa al artículo 32 de la CRE en el cual manifiesta que el Estado debe garantizar el acceso a la salud mediante políticas económicas, sociales, culturales, educativas y ambientales, y se regirá por los principio de equidad, universalidad, solidaridad, interculturalidad, calidad, eficiencia, eficacia.

A sí mismo, en la investigación se reflejó que se vulnera el derecho a la alimentación, misma que no es saludable, las porciones no son las correctas para una persona adulta, además no hay una planificación de una dieta saludable, dando como resultado la vulneración al derecho de alimentación que se encuentra en nuestra CRE en su artículo 13 el cual indica claramente que todas las personas tienen un derecho seguro al acceso de una alimentación sana, es decir el estado garantiza este derecho. 
También se identifica que no existe un buen trato en el centro de rehabilitación, debido al constante abuso de los guías con el pretexto de mantener el orden, provocando de esta manera no solo un maltrato físico si no también psicológico, tanto así que las PPL tienen temor a reclamar o denunciar por las represalias posteriores, de manera clara y directa vulnera el artículo 66 numeral 3 literal c en el cual se prohíbe cualquier clase de tortura.

En este sentido, las vulneraciones de los derechos humanos a las PPL se dan por una falta de control de las autoridades dejándoles desprotegidos a las PPL, sin duda es una obligación aplicar la normativa constitucional y las que se encuentran en los instrumentos internacionales de Derechos Humanos, al no aplicar dicha normativa se viola el artículo 426 de la CRE, que señala la aplicabilidad y cumplimiento inmediato de estas normativas.

En relación a lo investigado, si existiera un control y un trabajo adecuado de todos los funcionarios y autoridades se frenaría un buen porcentaje de las mafias existentes y las personas privadas de a libertad incluso podrían aportar con su trabajo a nuestra sociedad.

\section{FINANCIAMIENTO}

No monetario.

\section{AGRADECIMIENTO}

A las Autoridades del Centro de Rehabilitación Social Turi por prestar el apoyo para el desarrollo de la investigación. 


\section{REFERENCIAS CONSULTADAS}

Amnistía Internacional (2014). Derechos Humanos para la dignidad humana. [Human rights for human dignity]. Recuperado de https://n9.cl/m8mx

Asamblea General de las Naciones Unidas (1948). Declaración Universal de Derechos Humanos. [Declaration of Human Rights]. Recuperado de https://n9.cl/o2t5

Asamblea Mundial de la Salud (1946). Constitución de la Organización Mundial de la Salud. [Constitution of the World Health Organization]. Recuperado de https://n9.cl/y34t

Asamblea Nacional del Ecuador (2014). Código Orgánico Integral Penal. [Organic Comprehensive Criminal Code]. Recuperado de https://n9.cl/3k1u

Aldana-Zavala, J., \& Isea, J. (2018a). Derechos Humanos y Dignidad Humana. [Human Rights and Human Dignity]. IUSTITIA SOCIALIS, 3(4), 8-23. Recuperado de https://n9.cl/5uw27

Aldana-Zavala, J., \& Isea-Argüelles, J. (2018b). Derechos Humanos y Soberanía. [Human Rights and Sovereignty]. IUSTITIA SOCIALIS, 3(5), 29-48. http://dx.doi.org/10.35381/racji.v3i5.168

Baculima-Llivisaca, G., Narváez-Zurita, C., Trelles-Vicuña, D., \& Erazo-Álvarez, J. (2020). Derecho a la defensa en la detención con fines investigativos. [Right to defense in detention for investigative purposes]. IUSTITIA SOCIALIS, 5(8), 333352. http://dx.doi.org/10.35381/racji.v5i8.577

Barrera, E. (2015). La Salud en Colombia. Entre el Derecho a la salud y la Racionalidad Económica del Mercado 1993-2015. [Between the Right to health and the Economic Rationality of the Market 1993-2015]. Recuperado de https://n9.cl/g8hen

Behar-Rivero, D. (2018). Metodología de la Investigación. [Investigation methodology]. Recuperado de https://n9.cl/k9q2

Carretero-García, A. (2018). La Vulneración del Derecho Humano a la Alimentación. [The Violation of the Human Right to Food]. Recuperado de https://n9.cl/u3d7s

Casar-Hernández. (2008). Los Derechos Humanos y su Protección: Estudios sobre derechos humanos y derechos fundamentales. [Human Rights and their Protection: Studies on human rights and fundamental rights]. Recuperado de https://n9.cl//x9yp 
Constitución de la República del Ecuador (2008). Título III. Garantías Constitucionales. [Title III. Constitutional guarantees. Recuperado de https://n9.cl/sia

Corte Constitucional del Ecuador (2019). Caso N. ${ }^{\circ} 209-15-\mathrm{JH}$ y 359-18-JH. [Case No. 209-15-JH and 359-18-JH] Recuperado de https://n9.cl//fp8b

Corte Interamericana de Derechos Humanos (2020). Cuadernillo de jurisprudencia № 9: Personas Privadas de la Libertad. [Case law booklet No. 9: Persons Deprived of Liberty]. Recuperado de https://n9.cl/v2df

Erazo-Álvarez, J. C., \& Narváez-Zurita, C. I. (2020). Medición y gestión del capital intelectual en la industria del cuero - calzado en Ecuador. [Measurement and management of intellectual capital in the leather industry - footwear in Ecuador]. Revista Arbitrada Interdisciplinaria Koinonía, 9(5), 437-467. http://dx.doi.org/10.35381/r.k.v5i9.662

El Universo (2020). Sentencia a policias que golpearon en 2016 a presos de la cárcel de Turi. [Sentence to police officers who beat prisoners of the Turi prison in 2016] Recuperado de https://url2.cl/tryUz

El Telégrafo (2018). La calidad de la comida de los presos, bajo observación. [The food quality of the prisoners, under observation]. Recuperado de https://n9.cl/4o8z

Ferrajoli, L. (2001). Los Fundamentos de los Derechos Fundamentales. [The Fundamentals of Fundamental Rights]. Madrid: Editorial Trotta.

Hernández, R., Fernández, C., \& Baptista, M. (2014). Metodología de la Investigación. [Research Methodology]. Mexico DF: McGraw-Hill / Interamericana Editores, S.A. DE C.V.

INREDH (2018). Violación de derechos humanos en el CDP de Quito y la cárcel Turi. [Violation of human rights in the CDP of Quito and the Turi prison]. Recuperado de https://url2.cl/26cmR

Ministerio de Justicia Derechos Humanos y Cultos (2016). Reglamento del Sistema Nacional de Rehabilitación Social del Ministerio de Justicia, Derechos Humanos y Cultos. [Regulation of the National System of Social Rehabilitation of the Ministry of Justice, Human Rights and Worship]. Recuperado de https://n9.cl/mzle

Naciones Unidas (1976). Pacto Internacional de Derechos Civiles y Políticos. [International Covenant on Civil and Political Rights]. Recuperado de https://n9.cl/rs8b 
Naciones Unidas. (1976). Pacto Internacional de Derechos Económicos, Sociales y Culturales. [International Covenant on Economic, Social and Cultural Rights]. Recuperado de https://n9.cl/51ajs

Naciones Unidas (1987). Convención contra la Tortura y Otros Tratos o Penas Crueles, Inhumanos o Degradantes. [Convention against Torture and Other Cruel, Inhuman or Degrading Treatment or Punishment]. Recuperado de https://n9.cl/h7xc2

Organismo Técnico de Rehabilitación Social (2018). Reglamento para el funcionamiento del Organismo Técnico del sistema nacional de Rehabilitación. [Regulations for the operation of the Technical Organism of the national Rehabilitation system]. Recuperado de https://n9.cl/leap

Pacheco, J. A. (2016). El derecho a la salud en las personas privadas de la libertad: el caso de un establecimiento penitenciario y carcelario en el departamento de Antioquia, 2014. [The Right to Health of Persons Deprived of their Liberty: The case of a penitentiary and Prision in Antioquia - 2014]. Recuperado de https://n9.cl/u1yd

Presidencia de la República del Ecuador (2014). Decreto Ejecutivo 365. [Executive Decree 365]. Recuperado de https://n9.cl/8tij

Presidencia de la República del Ecuador (2008). Decreto ejecutivo 1317. [Executive decree 1317]. Recuperado de https://n9.cl/4r32s

Presidente Constitucional Interino de la República (1998). Plan Nacional de Derechos Humanos en el Ecuador. [National Plan for Human Rights in Ecuador]. Recuperado de https://n9.cl/jo9t

Reyes-Idrovo, M. G., Narváez-Zurita, C. I., Pozo-Cabrera, E. E., \& Erazo-Álvarez, J. C. (2020). La Educación Superior en el Ecuador: Derecho o servicio. [Higher Education in Ecuador: Law or service]. Iustitia Socialis, 1(5), 4-21. http://dx.doi.org/10.35381/racji.v5i1.598

Rodas-Cordero, D. C., Erazo-Álvarez, J. C., Pinos-Jaén, C. E., \& Narváez-Zurita, C. I. (2020). Derecho a Resistencia en el Marco Constitucional Ecuatoriano, aplicación del Principio Democrático y Seguridad Jurídica. [Right to Resistance in the Ecuadorian Constitutional Framework, application of the Democratic Principle and Legal Security]. Iustitia Socialis, 1(5), 78-100. http://dx.doi.org/10.35381/racji.v5i1.603 


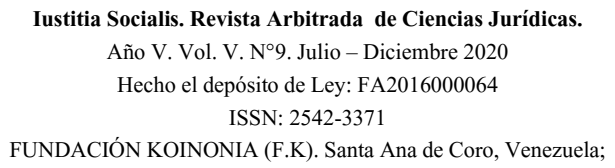

Tatiana Daniela Gárate-Osorio; Juan Carlos Erazo-Álvarez; Cecilia Ivonne Narváez-Zurita; Camilo Emanuel Pinos-Jaén

Salinas-Herrera, F. M., Narváez-Zurita, C. I., Vázquez-Calle, J. L., \& Erazo-Álvarez, J. C. (2020). Las medidas cautelares en materia constitucional en el Ecuador. [Precautionary measures in constitutional matters in Ecuador]. Iustitia Socialis, 1(5), 660-677. http://dx.doi.org/10.35381/racii.v5i1.635

Stieppel, J. (2013). Cárcel, Derecho y Politica. [Jail, Law and Politics]. Reduperado de https://n9.cl/uvvo

Tribunal de garantías penales con sede en el cantón Cuenca (2019). Juicio $\mathrm{N}^{\circ}$ 01283201604915G. [Judgment No. 01283201604915G]. Reduperado de https://n9.cl/n1jcz 